\title{
RADIO-SOURCE SPECTRA AND THEIR TIME VARIATIONS
}

\author{
K. I. Kellermann and I. I. K. Pauliny-Toth \\ (National Radio Astronomy Observatory, Green Bank, W.V., U.S.A.)
}

During the past few years there has been a large increase in the available data on the spectra of radio sources, particularly at short wavelengths, where a number of sources have shown unexpectedly large time variations, with time-scales of 1 year or less.

The simple power-law spectrum, which is a straight line on a log-log plot of flux density against frequency, is shown by about $30 \%$ of sources. Most sources have a spectrum with negative curvature, which steepens at high frequencies. Many have a sharp cut-off, which is almost certainly due to synchrotron self-absorption, at low frequencies. In several of these sources, such as 3C 48, 3C 147 and 3C 295, the spectrum begins to flatten at a considerably higher frequency than the cut-off frequency. This flattening is too sharp to be caused by a change in the energy distribution of the electrons and is probably due to parts of the source becoming optically thick at higher frequencies. Some sources have components which are optically thick even at centimetre wavelengths. These must have angular sizes of $10^{-3 \prime}$ or less. The energy density in relativistic electrons in these compact sources is much larger than the magnetic-energy density, so that the source cannot be stable and variations in the flux density are to be expected.

A total of some 24 variable radio sources are now known, including 13 QSS and 3 galaxies. The QSS for which data are available are known to show variations at optical wavelengths, these being more rapid and more violent than the radio variations. All of the variable sources have radio components of very small angular size and their spectra indicate that these components are optically thick near the wavelength where the flux is increasing and optically thin in the region where the flux is decreasing. This is consistent with the model of a variable component which is initially optically thick, but which becomes optically thin at successively longer wavelengths owing to its expansion. The flux density at a particular frequency thus increases until the source is optically thin at which time it begins to decrease. A characteristic age for this component may be estimated from the observed rate of change of flux, from the maximum flux density reached at different frequencies or from the times at which these maxima are reached. At centimetre wavelengths the characteristic time-scale is about 1 year. The required input of energy in the form of relativistic electrons is about $10^{58}$ ergs if the QSS are at cosmological distances, and this energy has to be released in a violent event lasting only a few months. Some sources, such as

Perek (ed.), Highlights of Astronomy, 371-372. (c) I.A.U. 
3C 273 and 3C 279, have complex spectra indicating the simultaneous presence of two or three variable components with ages of 1-10 years. Since about $10 \%$ of the QSS are currently active, it seems that in the typical QSS there occur periodic explosions every $1-100$ years, each with an energy output in particles of about $10^{8}$ Type-II supernovae. Each such explosion gives rise to a temporary increase in the radiated flux and a radio spectrum with positive curvature. During the dormant period, or after the cessation of all activity, the combined remnants of many explosions will give rise to the 'normal' power-law or slightly negatively curved spectrum.

If we consider only that part of the spectrum where the sources are optically thin, then all sources have similar spectral indices. At short wavelengths most radio galaxies are optically thin and the spectral indices are concentrated near -0.8 . A few galaxies with flatter spectra are Seyfert or Type $\mathbf{N}$ galaxies with active nuclei. The QSS have a broader distribution of spectral indices and a larger proportion have flat spectra. This is probably due entirely to self-absorption. QSS selected from lowfrequency surveys tend to be optically thin down to these frequencies and have 'normal' spectra. In their linear dimensions and radio properties they tend to be similar to the radio galaxies. QSS selected from surveys at higher frequencies have a greater proportion of sources which are optically thick, which are always very small, often variable and intrinsically more luminous than the sources with steeper spectra.

\section{DISCUSSION}

J.A. Roberts: Is there any change of angular size with wavelength in the case of sources with peculiar spectra?

Kellermann: Yes. In 3C 279, for example, the angular diameter is $0.01^{\prime \prime}$ of arc at centimetric wavelengths but $2^{\prime \prime}-3^{\prime \prime}$ of arc at metre wavelengths.

Sturrock: Have you considered that the presence of dense plasma might affect the time-scale of the high-frequency variations?

Kellermann: There is unlikely to be such plasma because of the negligible depolarization of the radio emission.

Scheuer: Another argument against such plasma is that the free-free absorption in it would extend to a frequency of many gigahertz. 